\title{
AN ANNOTATED LIST OF THE LICHENS AND LICHENICOLOUS FUNGI OF THE TETERIV RIVER BASIN (UKRAINE)
}

\author{
NADIIA KAPETS
}

\begin{abstract}
First data on lichens of the Teteriv River Basin appeared in the second part of 19th century. First records of lichenicolous fungi in this area were published at the end of the 20th century. An annotated list of lichens and lichenophilous fungi is based on literature data and materials of own researche of lichenobiota of the upper and middle course of the Teteriv river basin. Llist of lichens and lichenicolous fungi of the area includes 300 species. The distribution of 117 species (80 lichens, 37 lichenicolous fungi) are for the first time recorded for area studied. Five species (Cercidospora macrospora, Lichenostigma epipolina, Lichenothelia tenuissima, Polysporina subfuscescens i Taeniolella beschiana) are new to Ukraine, 7 species (Anisomeridium polypori, Coenogonium pineti, Lichenoconium usneae, Monodictys epilepraria, Rinodina efflorescens, Sphaerellothecium propinquellum, Taeniolella punctata) are new for the Ukrainian Plains, while 11 species (Cornutispora lichenicola, Erythricium aurantiacum, Heterocephalacria physciacearum, Intralichen christiansenii, Laetisaria lichenicola, Lichenothelia convexa, Marchandiomyces corallinus, Psoroglaena dictyospora, Pyrenochaeta xanthoriae, Sarcogynelapponica, Scoliciosporum gallurae, Stigmidium xanthoparmeliarum) are new for the Polissia subprovince of mixed coniferous and broadleaved forests, and one species is new for the territory of the Ukrainian forest-steppe subprovince. In addition, new information on the distribution of a number of rare to Ukraine lichen and lichenicolous fungal species (Absconditella lignicola, Cornutispora lichenicola, Clypeococcum hypocenomycis, Lichenochora obscuroides, Lichenodiplis lecanorae, Marchandiomyces corallinus, Psoroglaena dictyospora, Sclerococcum sphaerale, Strangospora deplanata, Thelocarpon epibolum ect.) is obtained.
\end{abstract}

Keywords: lichens, lichenicolous fungi, lichenobiota, rare species, Teteriv River, Ukraine.

\section{INTRODUCTION}

First data on lichens of the Teteriv River Basin appeared in the second part of 19th century [4]. First records of lichenicolous fungi in this area were published at the end of the 20th century [12]. Before our research 181 species of lichens, one lichenicolous fungus (Trichonectria hirta (A. Bloxam) Petch) and one epilithic fungus (Lichenothelia scopularia (Nyl.) D. Hawksw.) were known in the area. We got an interesting data on lichenobiota of the territory. As a result 117 species are recorded at the first time from the Teteriv River Basin, from them 80 lichens and 37 lichenicolous fungi. An annotated list of species is provided in this paper. 


\section{Research Objective, Methodology AND DATA}

The Teteriv River flows in the north of Ukraine. It is a right-bank tributary of the Dniper River. The Teteriv River has an overall length of $365 \mathrm{~km}$, a depth of about $1.0-1.5 \mathrm{~m}$ and basin of $15,100 \mathrm{~km}^{2}$. The Teteriv River Basin is located in the northwestern part of the Ukrainian Crystal Shield, which is largely affecting on natural conditions of the area. Rivers Hnylop'yat, Huyva, Zdvyzh and Irsha are the major tributaries of the Teteriv. The main type of land-scape of the area are outwash plains with a crystalline basement. The main types of soils are turf-podzolic, turf-podzol, black and forest soils. River banks are steep, usually to 1-2 m high and often with granite outcrops form coastal rocks. Granite outcrops also form rapids, coastal cliffs, canyons of 25-30 m tall. Most granite outcrops are located at the upper and middle course of the Teteriv River Basin [13].

The climate is temperate continental. Continuity of the climate increases in the direction from northwest to southeast. The average annual temperature is $6-7{ }^{\circ} \mathrm{C}$. Average temperature of July is $19.4^{\circ} \mathrm{C}$, of January is $-5.7^{\circ} \mathrm{C}$. Average annual precipitation reaches about $562 \mathrm{~mm}$ [14].

The middle and lower courses are located in the forest zone of Ukraine and only the small part of upper course of the Teteriv River Basin is located in the forest-steppe zone of Ukraine. The predominant type of vegetation is forest vegetation accounted for more than half of the area. There are three main types of forest vegetation: 1) basiphilous beech and mixed fir-beech forests; 2) acidophilous oak and oak-birch forests on nutrient-poor soils; and 3) pine forests on nutrient-poor and hydromorphic soils. The distinctive feature of this area is a presence of chasmophytic vegetation of crevices, rocky ledges and faces of rocky cliffs. Ombrophilous lichen communities of siliceous rock surfaces are spread on outcrops of the Crystal Shield and presented mostly by communities of Rhizocarpetea geographici Wirth 1972. Pioneer vegetation on shallow soils on rocky siliceous outcrops on siliceous rocks are common for the whole territory $[2,7,15]$.

The data on lichens and lichenicolous fungi were obtained the 2013-2020 within the framework of inventory of lichenobiota of the upper and middle course of the Teteriv river basin Teteriv River Basin. The material was collected using the classic methods of a field sampling. All specimens were identified in laboratory using light microscope [12], special chemicals and modern literature [1, 3, 5, 12]. The nomenclature of taxa of lichenicolous fungi followed the Index Fungorum. The main collected samples of lichenicolous fungi are deposited in the Lichen herbarium of Ukraine (KW-L).

\section{RESULTS AND DISCUSSION}

An Annotated List of the Lichens and Lichenicolous Fungi of the Teteriv River Basin is based on published records and own research of the upper and middle course of the river basin. There are 300 species (261 lichens, 39 lichenicolous fungi) 125 genera, 52 families, 28 orders, 11 classes and 2 divisions. Totally 117 taxa (80 lichens, 37 lichenicolous fungi) newly recorded for the area. Five species (Cercidospora macrospora, Lichenostigma epipolina, Lichenothelia tenuissima, Polysporina subfuscescens and Taeniolella beschiana) are reported from Ukraine for the first time, 7 species (Anisomeridium polypori, Coenogonium pineti, Lichenoconium usneae, Monodictys epilepraria, Rinodina efflorescens, Sphaerellothecium propinquellum, Taeniolella punctata) are new for the Ukrainian Plains, while 11 species (Cornutisporalichenicola, Erythricium aurantiacum, Heterocephalacria physciacearum, Intralichen christiansenii, Laetisaria lichenicola, Lichenothelia convexa, Marchandiomyces corallinus, Psoroglaena dictyospora, Pyrenochaetaxanthoriae, Sarcogyne lapponica, Scoliciosporum gallurae, Stigmidium xanthoparmeliarum) are new for the Polissia subprovince of mixed coniferous and broad-leaved forests, and one (Pronectria leptaleae) species is new for the territory of the Ukrainian forest-steppe subprovince. In addition, new information on the distribution of a number of rare to Ukraine lichen and lichenicolous fungal species (Absconditella lignicola, Cornutispora lichenicola, Clypeococcum hypocenomycis, Lichenochora obscuroides, 
Lichenodiplis lecanorae, Marchandiomyces corallinus, Psoroglaena dictyospora, Sclerococcum sphaerale, Strangospora deplanata, Thelocarpon epibolum ect.) is obtained.

\section{List of species}

$!=$ Taxa added as new for the area.

$\mathrm{LF}=$ Lichenicolous fungi.

!Abrothallus caerulescens I. Kotte LF

!Absconditella lignicola Vězda \& Pišút

Acarospora fuscata (Nyl.) Th. Fr.

!Acarospora oligospora v. pachnea $\mathrm{H}$. Magn.

Acarospora veronensis A. Massal.

!Acrocordia gemmata (Ach.) A. Massal.

!Ainoa mooreana (Carroll) Lumbsch \& I. Schmitt

Alyxoria varia (Pers.) Ertz \& Tehler

Amandinea punctata (Hoffm.) Coppins \& Scheid.

Anaptychia ciliaris (L.) Körb. ex A. Massal.

!Anisomeridium polypori (Ellis \& Everh.) M.E. Barr

!Reichlingia anombrophila (Coppins \& P. James) Frisch

Arthonia dispersa (Schrad.) Nyl., Lich. Scand.

!Arthonia epiphyscia Nyl. LF

!Arthonia phaeophysciae Grube et Matzer LF

Arthonia radiata (Pers.) Ach., $\mathrm{K}$.

!Arthothelium lirellans (Almq.) Coppins

Aspicilia albomarginata B. de Lesd.

Aspicilia cinerea (L.) Körb.

Aspicilia reticulata Kremp.

Athallia cerinella (Nyl.) Arup

Athallia holocarpa (Hoffm.) Arup

Athallia pyracea (Ach.) Arup

!Athelia arachnoidea (Berk.) Jülich, Willdenowia LF

Bacidia bagliettoana (A. Massal. \& De Not.) Jatta

Bacidia phacodes Körb.

!Bellemerea cupreoatra (Nyl.) Clauzade \& Cl. Roux

!Biatora globulosa (Flörke) Fr.

!Blastenia crenularia (With.) Arup, Søchting \& Frödén

!Bryostigma muscigenum (Th. Fr.) Frisch \& G. Thor

Buellia badia (Fr.) A. Massal.

Buellia schaereri De Not.

Calicium lenticulare Ach.

Calicium trabinellum (Ach.) Ach.

Calogaya decipiens (Arnold) Arup

Calogaya pusilla (A. Massal.) Arup, Frödén \& Søchting

Calogaya saxicola (Hoffm.) Vondrák

Caloplaca cerina (Hedw.) Th. Fr. var. cerina

Candelaria concolor (Dicks.) Arnold

!Candelariella aurella (Hoffm.) Zahlbr.

!Candelariella coralliza (Nyl.) H. Magn.

!Candelariella reflexa (Nyl.) Lettau

Candelariella vitellina (Hoffm.) Müll. Arg.

Candelariella xanthostigma (Pers. ex Ach.) Lettau 
!Catillaria chalybeia (Borrer) A. Massal.

!Catillaria nigroclavata (Nyl.) J. Steiner

!Cercidospora crozalsiana (H. Olivier) Nav.-Ros., Cl. Roux \& Casares, Cryptog. Bryol.-

Lichénol. 16(2): 100 (1995) LF

!Cercidospora macrospora (Uloth) Hafellner \& Nav.-Ros., Lichen Flora of the Greater Sonoran

Desert Region (Tempe) 2: 638 (2004) LF

Cetraria sepincola (Ehrh.) Ach.

Chaenotheca ferruginea (Turner) Mig.

Chaenotheca furfuracea (L.) Tibell

!Chaenotheca stemonea (Ach.) Müll. Arg.

!Chaenotheca trichialis (Ach.) Hellb.

!Circinaria calcarea (L.) A. Nordin, Savić \& Tibell

Circinaria contorta (Hoffm.) A. Nordin

Cladonia arbuscula (Wallr.) Flot. subsp. arbuscula

!Cladonia bacilliformis (Nyl.) Sarnth.

Cladonia botrytes (K.G. Hagen) Willd.

!Cladonia cariosa (Ach.) Spreng.

!Cladonia carneola (Fr.) Fr.

Cladonia cenotea var. crassota $\mathrm{Nyl}$.

Cladonia cervicornis (Ach.) Flot. subsp. cervicornis

Cladonia chlorophaea (Flörke ex Sommerf.) Spreng.

Cladonia coccifera (L.) Willd.

Cladonia coniocraea (Flörke) Spreng.

Cladonia cornuta (L.) Hoffm.

Cladonia crispata (Ach.) Flot.

!Cladonia cyanipes (Sommerf.) Nyl.

Cladonia deformis (L.) Hoffm.

Cladonia fimbriata (L.) Fr.

Cladonia floerkeana (Fr.) Flörke

Cladonia furcata (Huds.) Schrad. subsp. furcata

!Cladonia glauca Flörke

Cladonia gracilis (L.) Willd.

!Cladonia grayi G. Merr. ex Sandst.

Cladonia macilenta Hoffm.

Cladonia mitis Sandst.

!Cladonia ochrochlora Flörke

Cladonia parasitica (Hoffm.) Hoffm.

Cladonia phyllophora Ehrh. ex Hoffm.

Cladonia pleurota (Flörke) Schaer.

!Cladonia pocillum (Ach.) O.J. Rich.

!Cladonia portentosa (Dufour) Coem.

Cladonia pyxidata (L.) Hoffm.

!Cladonia ramulosa (With.) J.R. Laundon

Cladonia rangiferina (L.) Weber ex F.H. Wigg.

Cladonia rangiformis Hoffm.

Cladonia rei Schaer.

!Cladonia scabriuscula (Delise) Leight.

Cladonia squamosa (Scop.) Hoffm.

Cladonia subulata (L.) Weber ex F.H. Wigg. 
Cladonia turgida Ehrh. ex Hoffm.

Cladonia uncialis (L.) Weber ex F.H. Wigg.

!Clypeococcum hypocenomycis D. Hawksw. LF

!Coenogonium pineti (Ach.) Lücking \& Lumbsch

Collema flaccidum (Ach.) Ach.

!Cornutispora lichenicola D. Hawksw. \& B. Sutton LF

Dermatocarpon miniatum (L.) W. Mann var. miniatum

Diploschistes scruposus (Schreb.) Norman

!Erythricium aurantiacum (Lasch) D. Hawksw. \& A. Henrici LF

Evernia divaricata (L.) Ach.

Evernia mesomorpha Nyl.

Evernia prunastri (L.) Ach.

Flavoparmelia caperata (L.) Hale

Flavoplaca citrina (Hoffm.) Arup, Frödén \& Søchting

!Fuscidea lygaea (Ach.) V. Wirth \& Vězda

Graphis scripta (L.) Ach.

Hertelidea botryosa (Fr.) Printzen \& Kantvilas

!Heterocephalacria physciacearum (Diederich) Millanes \& Wedin LF

Hypocenomyce scalaris (Ach. ex Lilj.) M. Choisy

Hypogymnia farinacea Zopf

Hypogymnia physodes (L.) Nyl.

Hypogymnia tubulosa (Schaer.) Hav.

Hypotrachyna revoluta (Flörke) Hale

Imshaugia aleurites (Ach.) S.L.F. Mey.

!Intralichen christiansenii (D. Hawksw.) D. Hawksw. \& M.S. Cole LF

Julella fallaciosa (Stizenb. ex Arnold) R.C. Harris

!Laetisaria lichenicola Diederich, Lawrey \& Van den Broeck LF

!Lecania cyrtella (Ach.) Th. Fr.

!Lecania cyrtellina (Nyl.) Sandst.

!Lecania dubitans (Nyl.) A.L. Sm.

!Lecania naegelii (Hepp) Diederich \& van den Boom

Lecanora albella (Pers.) Ach.

!Lecanora albellula (Nyl.) Th. Fr. var. albellula

!Lecanora allophana (Ach.) Nyl.

Lecanora alpigena (Ach.) $\mathrm{Cl}$. Roux

Lecanora argentata (Ach.) Röhl.

Lecanora carpinea (L.) Vain.

!Lecanora conizaeoides Nyl. ex Cromb.f. conizaeoides

Lecanora leptyrodes G.B.F. Nilsson

Lecanora lithophila Oxner

Lecanora populicola (DC.) Duby

Lecanora pulicaris (Pers.) Ach.

Lecanora rupicola (L.) Zahlbr. var. rupicola

!Lecanora saligna (Schrad.) Zahlbr.

Lecanora symmicta (Ach.) Ach.

Lecanora umbrina (Ach.) A. Massal.

!Lecanora varia (Hoffm.) Ach.

Lecidea fuscoatra (L.) Ach.

Lecidea lapicida (Ach.) Ach. 
Lecidea lithophila (Ach.) Ach.

Lecidella elaeochroma (Ach.) M. Choisy f. elaeochroma

!Lecidella euphorea (Flörke) Hertel

Lecidella stigmatea (Ach.) Hertel \& Leuckert

!Lendemeriella borealis (Vain.) S.Y. Kondr.

Lepra albescens (Huds.) Hafellner

Lepra amara (Ach.) Hafellner

!Lepraria elobata Tønsberg

Lepraria incana (L.) Ach.

!Lepraria lobificans Nyl.

Lepraria membranacea (Dicks.) Vain.

!Lichenochora obscuroides (Linds.) Triebel \& Rambold LF

!Lichenoconium erodens M.S. Christ. \& D. Hawksw. LF

!Lichenoconium lecanorae (Jaap) D. Hawksw. LF

!Lichenoconium usneae (Anzi) D. Hawksw. LF

!Lichenodiplis lecanorae (Vouaux) Dyko \& D. Hawksw. LF

!Lichenostigma cosmopolites Hafellner \& Calat. LF

!Lichenostigma epipolina Nav.-Ros., Calat. \& Hafellner LF

!Lichenothelia convexa Henssen LF

Lichenothelia scopularia (Nyl.) D. Hawksw.

!Lichenothelia tenuissima Henssen.

Lobaria pulmonaria (L.) Hoffm.

!Marchandiomyces corallinus (Roberge) Diederich \& D. Hawksw. LF

Melanelia subargentifera (Nyl.) Essl.

Melanelia subaurifera (Nyl.) Essl.

Melanelixia fuliginosa (Fr. ex Duby) O. Blanco, A. Crespo, Divakar, Essl., D. Hawksw. \&

Lumbsch

Melanelixia glabratula (Lamy) Sandler \& Arup

Melanohalea exasperata (De Not.) O. Blanco, A. Crespo, Divakar, Essl., D. Hawksw. \&

Lumbsch

Melanohalea exasperatula (Nyl.) O. Blanco, A. Crespo, Divakar, Essl., D. Hawksw. \& Lumbsch Melanohalea olivacea (L.) O. Blanco, A. Crespo, Divakar, Essl., D. Hawksw. \& Lumbsch !Micarea prasina Fr.

!Monodictys epilepraria Kukwa et Diederich LF

Montanelia sorediata (Ach.) Divakar, A. Crespo, Wedin \& Essl.

!Muellerella erratica (A. Massal.) Hafellner \& V. John LF

!Muellerella pygmaea (Körb.) D. Hawksw. LF

Nephroma parile (Ach.) Ach.

Nephromopsis chlorophylla (Willd.) Divakar, A. Crespo \& Lumbsch

Ochrolechia arborea (Kreyer) Almb.

Olegblumia demissa (Flot. ex Körb.) S.Y. Kondr., Lőkös, Jung Kim, A.S. Kondr., S.O. Oh \& Hur

Oxneria fallax (Arnold) S.Y. Kondr. \& Kärnefelt

!Pachyphiale fagicola (Arnold) Zwackh

Palicella filamentosa (Stirt.) Rodr. Flakus \& Printzen

Parmelia saxatilis (L.) Ach.

Parmelia sulcata Taylor

Parmelina tiliacea (Hoffm.) Hale

Parmotrema tinctorum (Despr. ex Nyl.) Hale

Peltigera canina (L.) Willd. 
Peltigera didactyla (With.) J.R. Laundon

Peltigera horizontalis (Huds.) Baumg.

Peltigera lepidophora (Vain.) Bitter

Peltigera malacea (Ach.) Funck

!Peltigera membranacea (Ach.) Nyl.

Peltigera polydactylon (Neck.) Hoffm.

Peltigera praetextata (Flörke ex Sommerf.) Zopf

Peltigera rufescens (Weis) Humb.

Peridiothelia fuliguncta (Norman) D. Hawksw.

Pertusaria coccodes (Ach.) Nyl.

!Pertusaria pertusa (L.) Tuck.

Phaeographis dendritica (Ach.) Müll. Arg.

Phaeophyscia ciliata (Hoffm.) Moberg

!Phaeophyscia endococcina (Körb.) Moberg

!Phaeophyscia hirsuta (Mereschk.) Essl.

!Phaeophyscia nigricans (Flörke) Moberg

Phaeophyscia orbicularis (Neck.) Moberg

!Phlyctis agelaea (Ach.) Flot.

!Phlyctis argena (Ach.) Flot.

Physcia adscendens H. Olivier

Physcia aipolia (Ehrh. ex Humb.) Fürnr.

Physcia caesia (Hoffm.) Hampe ex Fürnr.

Physcia dubia (Hoffm.) Lettau

Physcia stellaris (L.) Nyl.

Physcia tenella (Scop.) DC.

Physcia tribacia var. tribacia (Ach.) Nyl.

!Physconia detersa (Nyl.) Poelt

Physconia distorta (With.) J.R. Laundon

!Physconia enteroxantha (Nyl.) Poelt

Physconia grisea (Lam.) Poelt

!Physconia pulverulenta (Schreb.) Poelt

Placopyrenium trachyticum (Hazsl.) Breuss

Placynthiella dasaea (Stirt.) Tønsberg

!Placynthiella icmalea (Ach.) Coppins \& P. James

!Placynthiella oligotropha (J.R. Laundon) Coppins \& P. James

Placynthiella uliginosa (Schrad.) Coppins \& P. James

Platismatia glauca (L.) W.L. Culb. \& C.F. Culb.

Pleurosticta acetabulum (Neck.) Elix \& Lumbsch, in Lumbsch, Kothe \& Elix

Polycauliona candelaria (L.) Frödén, Arup \& Søchting,

Polycauliona polycarpa (Hoffm.) Frödén, Arup \& Søchting

Polyozosia dispersa (Pers.) S.Y. Kondr., L. Lókös \& Farkas

!Polyozosia hagenii (Ach.) S.Y. Kondr., L. Lőkös \& Farkas

!Polyozosia sambuci (Pers.) S.Y. Kondr., L. Lókös \& Farkas

Polysporina simplex (Taylor) Vězda

!Polysporina subfuscescens (Nyl.) K. Knudsen \& Kocourk. LF

!Pronectria leptaleae (J. Steiner) Lowen LF

Protoparmeliopsis muralis (Schreb.) M. Choisy

Pseudevernia furfuracea (L.) Zopf var. furfuracea

Pseudoschismatomma rufescens (Pers.) Ertz \& Tehler 
!Psoroglaena dictyospora (Orange) H. Harada

Punctelia subrudecta (Nyl.) Krog

!Pyrenochaeta xanthoriae Diederich LF

!Pyrenodesmia aractina (Fr.) S.Y. Kondr.

!Pyrenodesmia atroflava (Turner) S.Y. Kondr.

Ramalina baltica Lettau

!Ramalina calicaris (L.) Röhl.

!Ramalina dilacerata (Hoffm.) Hoffm.

Ramalina farinacea (L.) Ach.

Ramalina fraxinea (L.) Ach.

Ramalina pollinaria (Westr.) Ach.

Rhizocarpon distinctum Th. Fr.

Rhizocarpon eupetraeum (Nyl.) Arnold

Rhizocarpon geographicum (L.) DC.

Rhizocarpon grande (Flörke ex Flot.) Arnold

!Rhizocarpon lavatum (Ach.) Hazsl.

Rhizocarpon petraeum (Wulfen) A. Massal.

Rhymbocarpus neglectus (Vain.) Diederich \& Etayo

!Rinodina efflorescens Malme

!Rinodina exigua (Ach.) Gray

!Rinodina oxydata (A. Massal.) A. Massal.

!Rinodina pyrina (Ach.) Arnold

Rinodina teichophila (Nyl.) Arnold

!Sarcogyne lapponica (Ach. ex Schaer.) K. Knudsen \& Kocourk.

Sarcogyne privigna (Ach.) A. Massal.

!Sarcogyne regularis Körb.

!Sclerococcum sphaerale (Ach.) Fr. LF

Sclerophora pallida (Pers.) Y.J. Yao \& Spooner

Scoliciosporum chlorococcum (Graewe ex Stenh.) Vězda

!Scoliciosporum gallurae Vězda \& Poelt, in Nimis \& Poelt

!Scoliciosporum umbrinum (Ach.) Arnold

!Sphaerellothecium propinquellum (Nyl.) Cl. Roux \& Triebel LF

!Stigmidium fuscatae (Arnold) R. Sant. LF

!Stigmidium squamariae (B. de Lesd.) Cl. Roux et Triebel LF

!Stigmidium xanthoparmeliarum Hafellner LF

!Strangospora deplanata (Almq.) Clauzade \& Cl. Roux, Bull.

!Taeniolella beschiana Diederich LF

!Taeniolella phaeophysciae D. Hawksw. LF

!Taeniolella punctata M.S. Christ. \& D. Hawksw. LF

!Thelocarpon epibolum Nyl.

Trapelia coarctata (Turner) M. Choisy

Trapelia involuta (Taylor) Hertel

!Trapelia obtegens (Th. Fr.) Hertel, Vortr. GesGeb. Bot. 4: 181 (1970)

Trapeliopsis flexuosa (Fr.) Coppins \& P. James

!Trapeliopsis granulosa (Hoffm.) Lumbsch

!Trapeliopsis viridescens (Schrad.) Coppins \& P. James

Trichonectria hirta (A. Bloxam) Petch LF

Usnea ceratina Ach.

Usnea florida (L.) Weber ex F.H. Wigg. 
Usnea glabrescens (Nyl. ex Vain.) Vain.

Usnea hirta (L.) Weber ex F.H. Wigg.

Usnea subfloridana Stirt.

Verrucaria aethiobola Wahlenb.

Verrucaria nigrescens Pers. f. nigrescens

Vulpicida pinastri (Scop.) J.-E. Mattsson \& M.J. Lai

Xanthoparmelia conspersa (Ehrh. ex Ach.) Hale

Xanthoparmelia pulla (Ach.) O. Blanco, A. Crespo, Elix, D. Hawksw. \& Lumbsch

Xanthoparmelia somloënsis (Gyeln.) Hale, in Ahti, Brodo \& Noble

!Xanthoparmelia verrucella (Essl.) O. Blanco, A. Crespo, Elix, D. Hawksw. \& Lumbsch

!Xanthoria calcicola Oxner

!Xanthoria elegans (Link) Th. Fr.

Xanthoria parietina (L.) Th. Fr. var. parietina

Xanthoria polessica S.Y. Kondr. \& A.P. Yatsyna

!Xanthoriicola physciae (Kalchbr.) D. Hawksw. LF

\section{ACKNOWLEDGEMENTS}

I would like to express my great gratitude to my academic advisor Dr. S. Ya. Kondratyuk for his support and valuable guidance to me. Special thanks to my colleague Dr Mariia Zykova, Liudmyla Popova, Svitlana Nyporko, Haluna Redko, Tetiana Suprun and my family for their help during the research.

\section{REFERENCES}

[1] Calatayud V., Navarro-Rosines P., Hafellner J. A synopsis of Lichenostigma subgen. Lichenogramma (Arthoniales), with a key to the species. Mycol. Research., 106 (10) (2002), 1230-1242. doi.org: 10.1017/s095375620200655x

[2] Didukh Y.P., Fitsailo T.V., Korotchenko I.A., Iakushenko D.M., Pashkevych N.A. Biotopes of forests and forest-steppe zones of Ukraine. Ltd. "Makros", Kyiv, 2011. (in Ukrainian)

[3] Diederich P. New or interesting lichenicolous fungi 2. Taeniolella beschiana new pecies and Taeniolella serusiauxi new species (Hyphomycetes). Bull. Soc. Nat. Luxembourg, 93 (1992), 155-162.

[4] Fedorenko N.M., Kondratyuk S.Ya., Orlov O.O. Lichen-forming and lichenicolous fungi of Zhytomyr Oblast. Ruta, Volyn, Zhytomyr, 2006. (in Ukrainian)

[5] Hawksworth D.L. A key to the lichen-forming, parasitic, parasymbiotic and saprophytic fungi occurring on lichens in the British Isles. Lichenologist, 15 (1) (1983), 1-44. doi: 10.1016/s00071536(84)80239-9

[6] Henssen A. Lichenothelia, a genus of microfungi on rocks. Bibl. Lichenol, 25 (1987), 257-293.

[7] Kapets N.V., Barsukov O.O., Vynokurov D.S., Khomyak I.V. Pioneer lichen communities of the Teteriv River Basin (Ukraine). Acta Botanica Hungarica, 60 (3-4) (2018), 331-355. doi: 10.1556/034.60.2018.3-4.6

[8] Kapets N.V., Kondratyuk S.Y. New data on lichenicolous fungi of the Teteriv River Basin (Ukraine). Acta Botanica Hungarica, 61 (1-2) (2019), 45-54. doi: 10.1556/034.61.2019.1-2.6

[9] Kapets N.V., Pleskach L.Yu., Popova L.P., Fedorenko N.M., Litovynska, A.V., Shershova N.V., Kondratyuk S.Ya. New to Ukraine and rare species of lichenorming and lichenicolous fungi. Ukr. Bot. J., 72 (2) (2015), 156-163. doi: 10.15407/ukrbotj72.02.156 (in Ukrainian)

[10] Knudsen K., Kocourkova, J. A study of lichenicolous species of Polysporina (Acarosporaceae). Mycotaxon, 105 (2008), 149-164.

[11] Kondratyuk S.Ya., Martynenko V.G. Lichen indication (manual). TOV 'KOD', Kiev-Kirovograd, 2006. (in Ukrainian) 
[12] Kondratyuk S.Ya., Andrianova T.V., Tykhonenko Yu.Yu. Study of mycobiota diversity of Ukraine (lichenicolous, Septoria and Puccinia fungi). M. G. Kholodny Institute of Botany, Phytosociocentre, Kyiv, 1999. (in Ukrainian)

[13] Marinich A.M. The geomorphology of South Polessye. Publishing House of Kyiv State University, Kiev, 1963. (in Russian)

[14] Marinich A.M., Pashchenko V.M., Shyshchenko P.H. The nature of the Ukrainian SSR. Landscapes and physical-geographical oblastalization. Naukova Dumka, Kiev, 1985. (in Russian)

[15] Orlov O.O., Yakushenko D.M. Plant cover of projected Korostyshiv Nature National Park. Fitosotsiotsentr, Kyiv, 2005. (in Ukrainian)

Address: Nadiia Kapets, Vasyl Stefanyk Precarpathian National University, 201 Halytska St., IvanoFrankivsk 76018, Ukraine.

E-mail: kapets_n@ukr.net.

Received: 10.10 .2020 ; revised: 28.12 .2020 .

Капець Надія. Анотований писок дишайників та ліхенофільних грибів басейну річки Тетерів (Україна). Журнал Прикарпатського університету імені Василя Стефаника, 7 (4) (2020), 76-85.

Перші відомості про лишайники басейну річки Тетерів датуються другою половиною XIX ст., тоді як перша інформація про знахідки ліхенофільних грибів у межах цієї території з'явились лише наприкінці XX ст. У статті наводиться анотований список дишайників та ліхенофільни грибів, що грунтується на літературних даних та матеріалах власних досліджень ліхенобіоти верхньої та середньої течії басейну р. Тетерів. Анотований список вкдючає 300 видів (261 вид дишайників та 39 видів ліхенофільних грибів). Вперше для території досліджень вказується поширення 117 видів (80 видів лишайників та 37 видів ліхенофільних грибів). Вперше для України виявлено місцезростання 5 видів (Cercidospora macrospora, Lichenostigma epipolina, Lichenothelia tenuissima, Polysporina subfuscescens i Taeniolella beschiana), тоді як 7 видів (Anisomeridium polypori, Coenogonium pineti, Lichenoconium usneae, Monodictys epilepraria, Rinodina efflorescens, Sphaerellothecium propinquellum, Taeniolella punctata) наводяться вперше для ії рівнинної частини. У результаті досліджень вдалось суттєво доповненити відомості про поширення низки цікавих та рідкісних для України лишайників і піхенофільних грибів. Зокрема, знахідки 11 видів (Cornutispora lichenicola, Erythricium aurantiacum, Heterocephalacria physciacearum, Intralichen christiansenii, Laetisaria lichenicola, Lichenothelia convexa, Marchandiomyces corallinus, Psoroglaena dictyospora, Pyrenochaeta xanthoriae, Sarcogyne lapponica, Scoliciosporum gallurae, Stigmidium xanthoparmeliarum) є першими для території Поліської підпровінції хвойношироколистяних лісів, поширення ще 1 виду (Pronectria leptaleae) - наведено вперше для Української дісостепової підпровінції. Окрім того під час досліджень в межах басейну річкиТетерів виявлено нові місцезростання низки рідкісних для України видів (Absconditella lignicola, Cornutisporalichenicola, Clypeococcum hypocenomycis, Lichenochora obscuroides, Lichenodiplis lecanorae, Marchandiomyces corallinus, Psoroglaena dictyospora, Sclerococcum sphaerale, Strangospora deplanata, Thelocarpon epibolum ect.).

Кдючові слова: дишайники, ліхенофільні гриби, ліхенобіота, рідкісні види, басейн річки Тетерів, Україна. 